\title{
Selection of highly fertilization-competent bovine spermatozoa through adhesion to the Fallopian tube epithelium in vitro
}

\author{
R. Gualtieri and R. Talevi \\ Dipartimento di Biologia Evolutiva e Comparata, Università di Napoli 'Federico II', Via Mezzocannone 8, \\ 80134 Napoli, Italy
}

\begin{abstract}
Mammalian spermatozoa undergo a marked reduction in number during their journey through the female reproductive tract. One of the checkpoints in the selection of fertilizing spermatozoa may be the transient adhesion to the Fallopian tube epithelium, an event previously shown to play a key role in sperm storage. Bovine spermatozoa adhering to the Fallopian tube epithelium in vitro may be synchronously released by sulphated glycoconjugates. In the present study, experiments were designed to quantify the number of spermatozoa selected through adhesion, and to compare the zona pellucida (ZP) binding and fertilization competence of the initial sperm suspension versus the bound and unbound sperm subpopulations. Results showed that: (1) a fraction accounting for about $30 \%$ of the initial sperm suspension was selected by
\end{abstract}

in vitro adhesion to oviductal epithelial cell monolayers; (2) selected spermatozoa, collected after heparin-induced release, had a significantly superior ZP binding and fertilization competence (mean \pm SD: $110 \pm 28$ bound spermatozoa per oocyte; \% cleavage, mean \pm SEM: $89 \pm 4$ ) compared with both the initial sperm suspension $(45 \pm 10$ bound spermatozoa per oocyte, $P<0.001$; \% cleavage: $69 \pm 3, P<0.05)$ and the unselected subpopulation (30 \pm 4 bound spermatozoa per oocyte, $P<0.001$; \% cleavage: $58 \pm 3, P<0.01)$. These findings support the hypothesis that binding to oviductal cells is not only beneficial for sperm survival but also represents a crucial step for the selection of spermatozoa endowed with superior fertilization competence.

\section{Introduction}

In mammals, millions of spermatozoa are ejaculated into the female reproductive tract at coitus but only a few are able to reach the ampulla at the time of ovulation. This marked reduction in the number of spermatozoa may be correlated with the presence of control mechanisms within the female reproductive tract that act as checkpoints for the selection of fertilizing spermatozoa. During the journey through the female reproductive tract, spermatozoa first interact with the lower oviduct microenvironment and then with the cumulus-oocyte complexes (COCs) transported within the upper oviduct. In several species, the period from the onset of oestrus to ovulation covers several hours or even days (Hunter, 1988); therefore, spermatozoa reaching the oviduct at mating must maintain a fertilization competence until ovulation. Both in vivo and in vitro studies indicate that the oviduct provides a suitable environment that extends the fertile life of spermatozoa until unknown ovulation-associated signals allow sperm migration toward the ampulla, the site of fertilization (Smith and Yanagimachi, 1990; Hunter et al., 1991; Harper, 1994). In humans, similar mechanisms appear to

Email: gualtier@dgbm.unina.it operate to extend the period during which spermatozoa in the female reproductive tract are fertile (Ellington et al., 1998a, 1999). However, although in the last 10 years several studies have addressed the role of either oviductal secretions (Parrish et al., 1989; McNutt and Killian, 1991; Anderson and Killian, 1994; ljaz et al., 1994; McNutt et al., 1994; Abe et al., 1995a; Chian and Sirard, 1995; Chian et al., 1995; Grippo et al., 1995), specific glycoproteins (Abe et al., 1995b) or direct cell contact (Ellington et al., 1991; Pollard et al., 1991; Chian and Sirard, 1995; Lefebvre et al., 1995) in the maintenance of the fertilization competence of spermatozoa, it is becoming increasingly clear that adhesion to the oviduct also plays a key role in sperm selection. In fact, several reports in different species indicate that only spermatozoa characterized by intact acrosomes (Gualtieri and Talevi, 2000), an uncapacitated status (Thomas et al., 1995; Lefebvre and Suarez, 1996; Fazeli et al., 1999), superior morphology (Thomas et al., 1994) and normal chromatin structure (Ellington et al., 1999) can adhere to tubal epithelial cells in vitro. It has been suggested that the sperm-zona pellucida (ZP) interaction represents a further step in sperm selection. In fact, binding of spermatozoa to ZP selects spermatozoa endowed with progressive motility, normal morphology and chromatin structure (Hoshi et al., 1996), and may 
also discriminate against spermatozoa with numerical chromosomal aberrations (Van Dyk et al., 2000).

The wide diffusion of assisted reproductive biotechnologies has led to an increasing interest in the study of the natural processes involved in sperm selection. As current IVF protocols bypass several steps in the natural fertilization process, understanding the effects of the oviductal microenvironment on sperm physiology may improve methods for sperm selection in IVF technologies. Gualtieri and Talevi (2000) developed an in vitro spermoviductal cell co-culture system and a quantitative adhesion assay to study the role of cell adhesion in the maintenance of the fertilization competence of spermatozoa and sperm selection in cows. As mentioned above, however, although results of studies in several species indicate that adhesion to Fallopian tube epithelial cells selects a superior sperm subpopulation, until now it has not been possible to recover this subpopulation for further characterization or use in reproductive biotechnologies. Sulphated glycoconjugates, such as heparin, fucoidan and dextran sulphate, are powerful inducers of sperm release in vitro (Talevi and Gualtieri, 2001). As heparin-like glycoconjugates are present in the bovine oviductal fluid and their concentrations and capacitating activities change, being maximal at oestrus (Parrish et al., 1989), it is hypothesized that sulphated glycosaminoglycans act as signals to allow sperm release and therefore migration toward the upper oviduct at the time of ovulation (Talevi and Gualtieri, 2001). The possibility of synchronously inducing the release of spermatozoa bound to the bovine Fallopian tube in vitro has provided, for the first time, an opportunity to study the ability of these spermatozoa to fertilize.

In the present study, the fertilization and the ZP-binding competence of the sperm subpopulation adhering to Fallopian tube monolayers in vitro were compared with those of the sperm subpopulation unable to adhere as well as to the whole ejaculate of single bulls from which the subpopulations were recovered in vitro. The results indicate that oviductal monolayers cultured in vitro are able to select spermatozoa with superior fertilization and ZP-binding abilities.

\section{Materials and Methods}

\section{Chemicals}

Pig $\mathrm{FSH}$, equine $\mathrm{LH}, \beta$-oestradiol, BSA (fraction $\mathrm{V}$ and fraction $V$ fatty acid free), heparin (sodium salt, purified from pig intestinal mucosa, H3393), gelatin, Hoechst 33258 and 33342, hypotaurine, adrenalin, and penicillamine were obtained from Sigma Chemical Company (Milan). M199, fetal calf serum (FCS), gentamycin, fungizone, Hepes and sodium bicarbonate were obtained from Gibco (Milan). Glutaraldehyde was obtained from TAAB Laboratories (Rome) and Percoll was obtained from Amersham-Pharmacia (Milan).
Reagents and water for preparation of saline and culture media were all cell-culture tested.

\section{Oviduct monolayers}

Oviducts were collected from cows at the time of slaughter and transported to the laboratory in Dulbecco's PBS supplemented with $50 \mu \mathrm{g}$ gentamycin $\mathrm{ml}^{-1}$ at $4^{\circ} \mathrm{C}$. Laminae of epithelial cells, recovered from oviducts of single animals by squeezing, were cultured in M199 supplemented with $50 \mu$ g gentamycin $\mathrm{ml}^{-1}, 1 \mu \mathrm{g}$ fungizone $\mathrm{ml}^{-1}$, and $10 \%$ FCS, as described by Gualtieri and Talevi (2000). Bovine oviductal epithelial cells (BOEC) were cultured in $10 \mathrm{~cm}$ Petri dishes (Falcon; Becton Dickinson, Milan) for 24-48 h and then transferred into four-well tissue culture dishes (Nunc) with or without $12 \mathrm{~mm}$ gelatin-coated glass round coverslips on the well bottom. Fresh media changes were performed every $48 \mathrm{~h}$. Cell confluence on both gelatin-coated glass surfaces and plastic was attained in about 7-10 days. Monolayers were used within $24-48 \mathrm{~h}$ after attainment of cell confluence. Within each experiment, BOEC monolayers from a single individual were washed three times in modified Tyrode's albumin lactate pyruvate medium (sp-TALP; Parrish et al., 1988, modified as described by Paula-Lopes et al., 1998), and left in this medium until addition of spermatozoa (1-3 h).

\section{Sperm preparation}

Frozen bovine semen from three bulls $(0.5 \mathrm{ml}$ straws; approximately $50 \times 10^{6}$ spermatozoa per straw), obtained from Semen Italy (San Giuliano Saliceta, Modena), was used in all experiments. Straws were thawed in a water bath at $38^{\circ} \mathrm{C}$ for $30 \mathrm{~s}$ and then washed and stained at the same time in a discontinuous Percoll gradient, containing $10 \mu \mathrm{g}$ Hoechst $33342 \mathrm{ml}^{-1}$. The semen samples were deposited on the top of the Percoll layers, prepared by depositing $1 \mathrm{ml} 90 \%$ and $1 \mathrm{ml} \mathrm{45 \%}$ Percoll to form two layers in a $15 \mathrm{ml}$ centrifuge tube, and centrifuged for $30 \mathrm{~min}$ at $200 \mathrm{~g}$. The supernatant was removed and the pellet, resuspended in $300 \mu \mathrm{l}$ sp-TALP, was brought to $5 \mathrm{ml}$ with sp-TALP, centrifuged at $350 \mathrm{~g}$ for $10 \mathrm{~min}$, and resuspended in $300 \mu \mathrm{l} \mathrm{sp-TALP}$. Aliquots of recovered spermatozoa were assessed for concentration and percentage of motility using a haemocytometer on a microscope stage heated at $38.5^{\circ} \mathrm{C}$. Hoechst staining was omitted when spermatozoa were prepared for separation of the bound (BSS) and unbound sperm subpopulations (UBSS) and subsequent assessment of fertilization and ZP-binding competence.

\section{Quantitative analysis of sperm subpopulation selected by adhesion to oviductal monolayers}

Confluent monolayers cultured on gelatin-coated coverslips in four-well tissue culture dishes (Nunc) 
were inseminated with the same Hoechst-labelled sperm suspension (prepared as described above) at 0.1, 0.5, 1 and $5 \times 10^{6}$ motile spermatozoa per well in $0.75 \mathrm{ml}$ IVF-TALP for $1 \mathrm{~h}\left(38.5^{\circ} \mathrm{C}, 5 \% \mathrm{CO}_{2}\right.$ in air) to determine in single ejaculates the number of spermatozoa able to adhere to oviductal monolayers in vitro. At the end of coculture, $0.5 \mathrm{ml}$ culture supernatant containing UBSS was collected from each well and the remaining UBSS was removed by washing each well five times and the oviductal monolayers with adhering spermatozoa were fixed in glutaraldehyde $2.5 \%$ in PBS, for $1 \mathrm{~h}$ at room temperature $\left(20^{\circ} \mathrm{C}\right)$, extensively washed and mounted with the same buffer on a glass slide. UBSS collected after $1 \mathrm{~h}$ of coculture were assessed for number of spermatozoa and percentage of motility and then reinseminated on parallel monolayers at $0.3,0.5$ and $2.5 \times 10^{6}$ motile spermatozoa per well in $0.75 \mathrm{ml}$ IVF-TALP for $1 \mathrm{~h}\left(38.5^{\circ} \mathrm{C}, 5 \% \mathrm{CO}_{2}\right.$ in air) to investigate whether spermatozoa unable to bind oviductal monolayers after $1 \mathrm{~h}$ of co-culture may acquire this ability after further co-incubation. Cocultures were fixed as described above after UBSS removal. For each well, fields of $0.286 \mathrm{~mm}^{2}$ were acquired using a Zeiss Axioplan microscope equipped with phase-contrast, fluorescence and Nomarsky optics, by means of an Optronix camera and KS 300 software (Zeiss, Milan). The number of bound spermatozoa was determined by analysing 10 fields of $0.286 \mathrm{~mm}^{2}$ for each well.

\section{Oocyte collection and in vitro maturation}

Ovaries were collected at the time of slaughter and transported to the laboratory in Dulbecco's PBS supplemented with $50 \mu \mathrm{g}$ gentamycin $\mathrm{ml}^{-1}$ (Gibco) at $35^{\circ} \mathrm{C}$. COCs were then collected by aspiration of individual follicles with an 18-gauge needle in M199, $50 \mu \mathrm{g}$ gentamycin $\mathrm{ml}^{-1}, 1 \mu \mathrm{g}$ fungizone $\mathrm{ml}^{-1}$ and $10 \%$ FCS. COCs were matured in the same medium (10 COCs per $50 \mu \mathrm{l}$ under light paraffin oil) supplemented with $2 \mu \mathrm{g}$ oestradiol $\mathrm{ml}^{-1}, 0.1 \mathrm{iu} \mathrm{FSH} \mathrm{ml}{ }^{-1}$ and $10 \mathrm{iu} \mathrm{LH} \mathrm{ml}{ }^{-1}$ (Sigma) for $24 \mathrm{~h}$ at $38.5^{\circ} \mathrm{C}, 5 \% \mathrm{CO}_{2}$ in air. The selection of COCs matured in vitro was not performed before insemination. After extensive rinses in Hepes-TALP, COCs were transferred to IVF-TALP (Paula-Lopes et al., 1998) and inseminated in groups of 50 as described below.

\section{Selection of sperm subpopulations on oviductal monolayers}

Confluent monolayers cultured in four-well tissue culture dishes (Nunc) were inseminated with the same sperm suspension at 3-5 $\times 10^{6}$ motile spermatozoa per well in $0.75 \mathrm{ml}$ IVF-TALP for $1 \mathrm{~h}\left(38.5^{\circ} \mathrm{C}, 5 \% \mathrm{CO}_{2}\right.$ in air), to recover the sperm subpopulations able or unable to adhere to oviductal monolayers from single ejaculates. As a control the same sperm suspension was added to 12-well tissue culture dishes (Falcon; Becton Dickinson) in which cell culture inserts (PET, $3 \mu \mathrm{m}$ pore size) precluded the contact between spermatozoa and oviductal monolayers. At the end of co-culture, both the sperm suspension contained in the inserts and the supernatants containing UBSS were collected by each well and pooled separately. Monolayers with adhering spermatozoa were extensively washed to remove remaining UBSS, retained in $0.5 \mathrm{ml}$ IVF-TALP and then $50-100 \mu \mathrm{g}$ heparin $\mathrm{ml}^{-1}$ was added to induce the release of BSS (Talevi and Gualtieri, 2001). After $30 \mathrm{~min}$ of incubation at $38.5^{\circ} \mathrm{C}, 5 \% \mathrm{CO}_{2}$ in air, the whole medium was collected from wells and pooled. The control, UBSS and released BSS received the same dose of heparin at the same time.

\section{Fertilization competence of selected sperm subpopulations}

For fertilization, the control sperm suspension, as well as UBSS and released BSS, were used to inseminate groups of 50 COCs matured in vitro at $1 \times 10^{6}$ motile spermatozoa per well, in $500 \mu \mathrm{l}$ IVF-TALP containing $10 \mu \mathrm{g}$ heparin $\mathrm{ml}^{-1}$ and a mixture of penicillamine, hypotaurine and adrenalin (PHE-mix; Parrish et al., 1988) at final concentrations of 20,10 and $1 \mu \mathrm{mol}$, respectively. After $18-20 \mathrm{~h}$ of co-incubation at $38.5^{\circ} \mathrm{C}, 5 \% \mathrm{CO}_{2}$ in air, COCs were transferred in Hepes-TALP and vortexed to remove cumulus cells. Oocytes were collected, washed in SOFaa-BSA (Gardner et al., 1994) and incubated in groups of 30 in $50 \mu l$ drops of the same medium under pre-equilibrated light paraffin oil at $38.5^{\circ} \mathrm{C}, 5 \% \mathrm{CO}_{2}$ in air. Fertilization was scored by assessing cleavage at $72 \mathrm{~h}$ after insemination.

\section{Sperm-ZP binding ability}

COCs retrieved from ovaries of slaughtered animals through follicle aspiration were denuded by vortexing in Hepes-TALP and stored at $4{ }^{\circ} \mathrm{C}$ in hyperosmolar salt solution $\left(1.5 \mathrm{~mol} \mathrm{MgCl}_{2}, 0.1 \%\right.$ polyvinylpyrrolidone (PVP), 40 mmol Hepes, $\mathrm{pH}$ 7.4) until use. The spermZP binding assay was performed by removing denuded oocytes from the salt storage solution. The denuded oocytes were washed five times in IVF-TALP, placed in groups of 10 in $100 \mu \mathrm{l}$ drops of sperm suspension at $1 \times$ $10^{5} \mathrm{ml}^{-1}$ under light mineral oil at $38.5^{\circ} \mathrm{C}, 5 \% \mathrm{CO}_{2}$ in air, and incubated overnight. Oocytes were inseminated with initial sperm suspension, UBSS and released BSS prepared from single ejaculates as described above. At the end of the incubation, oocytes were rinsed five times in IVF-TALP using fine bored glass pipettes to dislodge loosely bound spermatozoa, fixed for $1 \mathrm{~h}$ at room temperature in $2.5 \%$ glutaraldehyde in PBS, washed in PBS, stained for 5 min with $1 \mu \mathrm{g}$ Hoechst $33342 \mathrm{ml}^{-1}$, and washed and mounted on glass slides. Oocytes were compressed under the coverslips to visualize all bound 

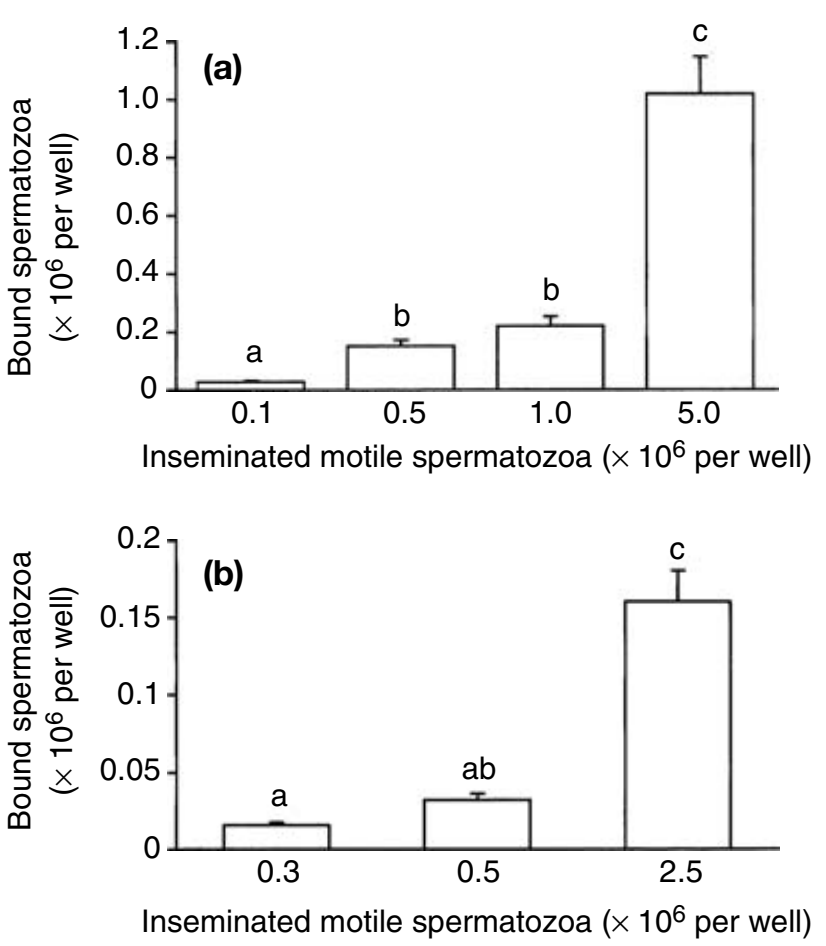

Fig. 1. (a) Effect of bovine sperm concentration on the number of spermatozoa bound to oviductal monolayers at $1 \mathrm{~h}$ of coincubation. Mean \pm SD bound spermatozoa per well; 0.1 versus $0.5, P<0.05 ; 0.5$ versus $1.0, P>0.05$; all other pairwise comparisons $P<0.001 ; n=6$. (b) Ability of reinseminated unbound sperm subpopulation to bind oviductal monolayers after $1 \mathrm{~h}$ of coincubation. Mean \pm SD bound spermatozoa per well; $P<0.001 ; 0.3$ versus $0.5, P>0.05 ; n=6$. Bars labelled with different letters indicate significant differences between the treatments $(P<0.05)$.

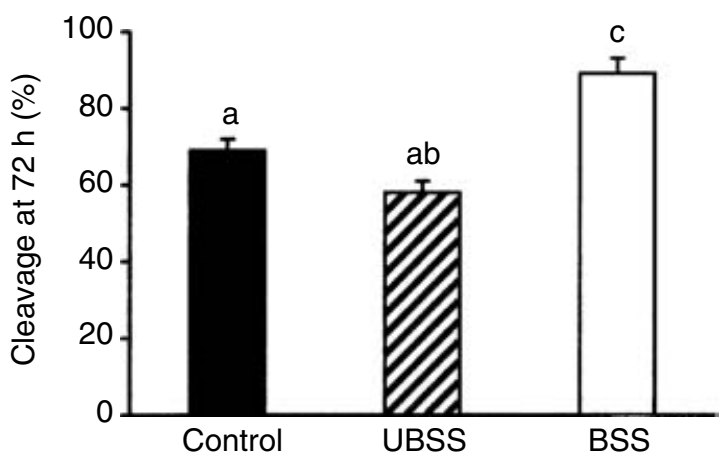

Fig. 2. Cleavage rates at $72 \mathrm{~h}$ after insemination of bovine cumulus-oocyte complexes fertilized with control spermatozoa, unbound sperm subpopulation (UBSS) and bound sperm subpopulation (BSS). BSS versus control, $P<0.05$; BSS versus UBSS, $P<0.01$; control versus UBSS, $P>0.05 ; n=4$. Bars labelled with different letters indicate significant differences between the treatments $(P<0.05)$.

spermatozoa in a single focus plane and images were acquired using a Zeiss Axioplan microscope equipped with fluorescence optics, by means of an Optronix camera and KS 300 software (Zeiss).

\section{Statistical analysis}

Data were analysed by one-way ANOVA (Systat 9; SPSS Inc.) followed by pair-wise comparison of means with Tukey's honestly significant difference. Data of fertilization competence were subjected to arc sin transformation before statistical analysis.

\section{Results}

Quantitative analysis of sperm subpopulation selected by adhesion

Spermatozoa collected and labelled by Hoechst staining and centrifugation on Percoll layers were inseminated on parallel monolayers at increasing sperm concentrations to determine the number of spermatozoa selected by adhesion to oviductal monolayers within $1 \mathrm{~h}$ of coincubation. Quantitative analysis showed that after $1 \mathrm{~h}$ of co-incubation, 20-30\% of inseminated spermatozoa were able to adhere to oviductal monolayers (Fig. 1a; motile spermatozoa inseminated $\times 10^{6}: 0.1$ versus 0.5 , $P<0.05 ; 0.5$ versus $1, P>0.05$; all other pairwise comparisons $P<0.001 ; n=6$ ). The remaining unbound spermatozoa comprising $70-75 \%$ of the initial sperm suspension were reinseminated on parallel monolayers and allowed to interact for $1 \mathrm{~h}$. Collected data showed that a small percentage (5-7\%) of this sperm population, which was unable to adhere to oviductal monolayers during the first hour of co-incubation, developed this ability during a second hour of co-culture (Fig. 1b; $P<$ $0.001 ; 0.3$ versus $0.5, P>0.05 ; n=6$ ).

\section{Fertilization competence of sperm subpopulations}

In vitro fertilization experiments were designed to investigate the fertilization competence of the single ejaculates and of subpopulations able or unable to adhere to bovine oviductal monolayers in vitro. Monolayers with adhering spermatozoa were treated with 50$100 \mu \mathrm{g}$ heparin $\mathrm{ml}^{-1}$ for $30 \mathrm{~min}$ to induce sperm release after collection of UBSS. As a control, the initial sperm suspension was co-cultured avoiding contact with the oviductal monolayers by means of cell culture inserts. The three sperm suspensions were used to inseminate groups of 50 COCs matured in vivo. Fertilization competence was expressed as the percentage of cleaved embryos at $72 \mathrm{~h}$ after insemination. Data indicate that the sperm subpopulation selected by $1 \mathrm{~h}$ of in vitro adhesion to oviductal monolayers had a superior fertilization ability (mean \pm SEM: $89 \pm 4 \%$ ) compared with the sperm subpopulation unable to adhere $(58 \pm 3 \%)$ as well as the initial sperm suspension from which UBSS and BSS were recovered (69 $\pm 3 \%$ ) (Fig. 2 ; BSS versus control, $P<$ 0.05; BSS versus UBSS, $P<0.01$; control versus UBSS, $P>0.05 ; n=4)$. 

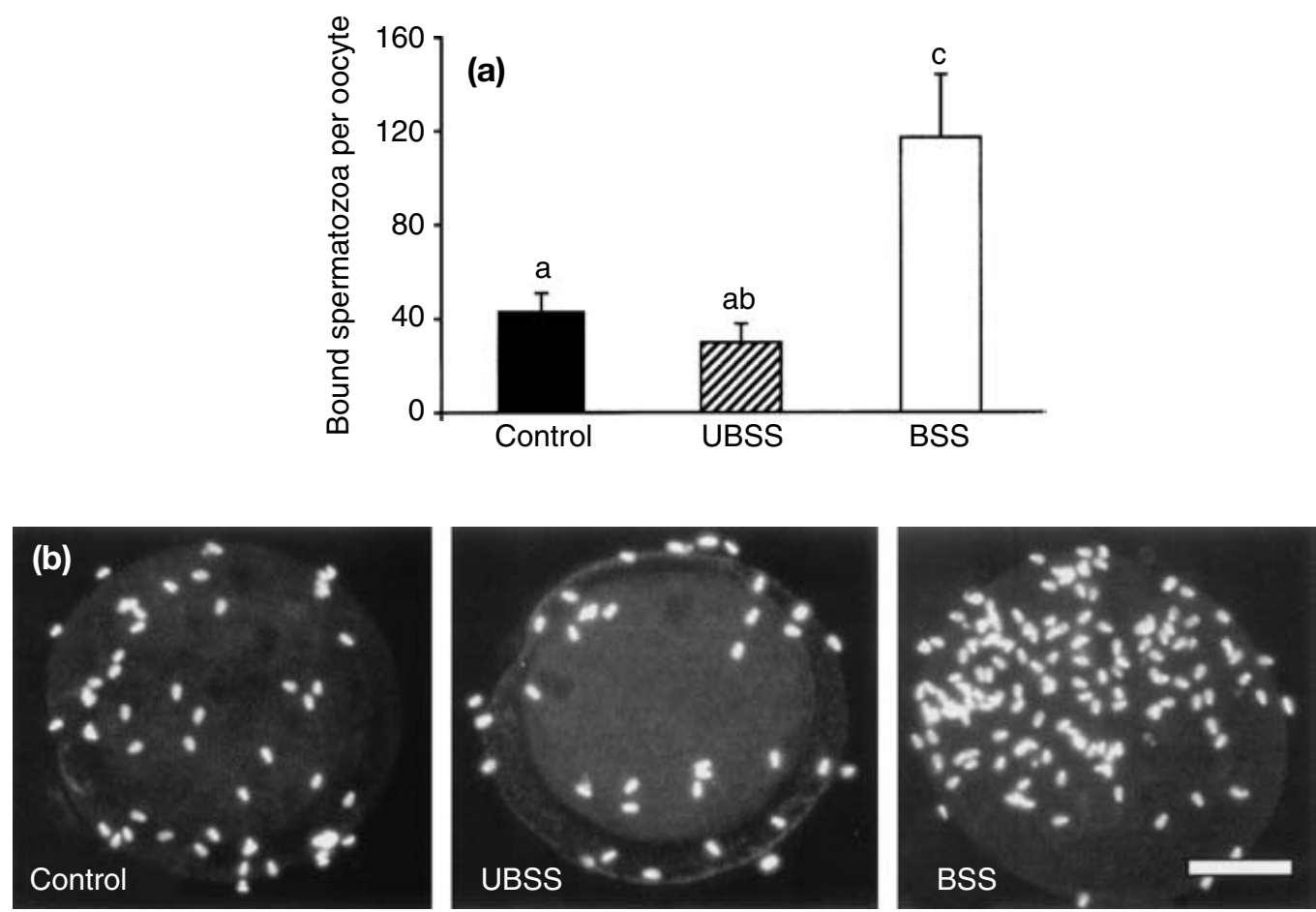

Fig. 3. Bovine sperm-zona pellucida-binding ability of control spermatozoa, unbound sperm subpopulation (UBSS) and bound sperm subpopulation (BSS). (a) Mean \pm SD bound spermatozoa per oocyte zona pellucida; control versus UBSS, $P>0.05$; all other pairwise comparisons $P<0.001 ; n=4$. (b) Fluorescence micrographs of salt-stored oocytes inseminated with control spermatozoa, UBSS and BSS. Scale bar represents $50 \mu \mathrm{m}$. Bars labelled with different letters indicate significant differences between the treatments $(P<0.05)$.

\section{Sperm-ZP binding ability}

The experiment was designed to investigate whether the initial sperm population and the two sperm subpopulations differed in their ability to bind the ZP of salt-stored oocytes. Results (control versus UBSS, $P>$ 0.05; all other pairwise comparisons $P<0.001 ; n=4)$ demonstrate that BSS (mean \pm SD; $117 \pm 27$ bound spermatozoa per oocyte) has a superior binding ability compared with both UBSS $(30 \pm 8)$ and the initial sperm population (42 \pm 9 ) (Fig. 3).

\section{Discussion}

The recent finding that sulphated glycoconjugates allow the synchronous release of spermatozoa bound to the bovine Fallopian tube in vitro (Talevi and Gualtieri, 2001) has provided the opportunity to investigate the fertilization competence of spermatozoa selected by adhesion to the oviductal epithelium. The main findings of the present study show that the sperm subpopulation able to adhere to Fallopian tube epithelium cultured in vitro has a higher fertilization competence compared with both the subpopulation unable to adhere and the initial ejaculate. These results demonstrate, for the first time in any species examined so far, that adhesion to oviduct epithelial cells is not only beneficial for prolonging the fertile lifespan of spermatozoa (Pollard et al., 1991), but also allows the selection of a pre-existing sperm subpopulation endowed with a higher fertilization or cleavage competence. These results highlight the selective function played by sperm-oviduct adhesion in the fertilization process. In fact, although several studies have shown that adhesion to the oviduct allows the selection of spermatozoa characterized by intact acrosomes (Gualtieri and Talevi, 2000), an uncapacitated status (Thomas et al., 1995; Lefebvre and Suarez 1996; Fazeli et al., 1999), low internal free calcium content and reduced tyrosine phosphorylation of membrane proteins (Petrunkina et al., 2001), superior morphology (Thomas et al., 1994), and normal chromatin structure (Ellington et al., 1999), until now it was impossible to determine whether these features of spermatozoa are associated with higher fertilization competence. Overall, the results of the present study indicate that the oviduct may have a dual role: to first select and then store particular sperm subpopulations.

In the present study, a subpopulation accounting for $30 \%$ of frozen-thawed Percoll recovered spermatozoa adhered to oviductal monolayers after $1 \mathrm{~h}$ of coincubation. Therefore, $70 \%$ of spermatozoa comprising the initial ejaculate were unable to adhere during this 
co-incubation time, indicating the presence of marked differences in the exposure of adhesion molecules of the sperm surface involved in the interaction with the oviduct. These differences may be attributable to the gradual acquisition of capacitation achieved by individual spermatozoa comprising the ejaculate. It has been suggested that rapid capacitation sperm-surface changes result in the induction of release operated by bull sperm capacitating agents (Talevi and Gualtieri, 2001). Capacitation markedly affects the ability of spermatozoa to bind oviductal epithelial cells in a number of species examined (Thomas et al., 1995; Lefebvre and Suarez 1996; Fazeli et al., 1999). The role played by capacitation in the ability of spermatozoa to bind the oviductal epithelium is confirmed by data from reinsemination experiments. In the present study, a $5-7 \%$ fraction of UBSS unable to bind oviductal monolayers at $1 \mathrm{~h}$ developed this ability during a further hour of co-culture, indicating that initially uncapacitated spermatozoa may undergo surface changes allowing sperm-oviduct adhesion. In this context, the unbound subpopulation may be highly heterogeneous, comprising both spermatozoa the surfaces of which are still coated by seminal plasma factors masking the molecules involved in oviduct adhesion, and capacitated spermatozoa that have lost the ability to bind to the oviduct. Although the capacitation status of the sperm subpopulations has not been characterized in the present study, it is possible that the ejaculate contains at least three different subpopulations: the first comprises spermatozoa still uncapacitated and unable to bind the oviductal epithelium; the second, able to bind, composed of spermatozoa that have already undergone the early steps of capacitation; and the third, unable to bind, in an advanced capacitation status. This would indicate that in the present study, oviduct adhesion selected most of the second subpopulation composed of early capacitated spermatozoa characterized by a superior fertilization competence compared with UBSS, mostly consisting of spermatozoa in an advanced capacitation state but also of a small fraction of uncapacitated spermatozoa.

The oviductal microenvironment exerts several positive effects on bovine gametes and embryos: (1) adhesion to the oviduct prolongs the fertile life of spermatozoa (Pollard et al., 1991); (2) oviductal fluid (Grippo et al., 1995) and media conditioned by oviduct epithelial cells (Chian and Sirard, 1995) improve the ability of spermatozoa to penetrate the oocyte; (3) a bovine oviductal specific glycoprotein, which is conserved in several mammals, increases the fertilization rate by an oocyte-specific effect (Verhage et al., 1997; Martus et al., 1998); and (4) embryo development is promoted by co-culture with oviductal cells (Eyestone and First, 1989; Ellington et al., 1990; Mermillod et al., 1993). However, until now the lack of a method to recover the spermatozoa adhering to the oviductal epithelium limited the analysis of the role played by the oviduct in sperm selection. In the present study, spermatozoa were exposed to oviductal monolayers for only $1 \mathrm{~h}$, a period sufficient to split the initial sperm suspension into two subpopulations, UBSS and BSS, whereas fertilization and embryo development were carried out in the absence of cultured cells. Moreover, to rule out the possible positive effects of secreted factors, oviduct monolayers were extensively washed with fresh medium before the addition of spermatozoa, and the control, that is the initial sperm suspension, was also co-cultured in inserts that avoided direct contact with the oviductal monolayers. Although there is a possibility that the enhanced fertilization competence of BSS also depends on the short period of adhesion with the oviductal monolayers, in the present study no declines were registered in the percentage of sperm motility and viability during the $1 \mathrm{~h}$ co-culture period either in control spermatozoa cultured in inserts or in UBSS compared with the initial sperm suspension (data not shown). Overall, such data demonstrate that a sperm subpopulation endowed with a superior fertilization or cleavage competence pre-exists within an ejaculate and can be selected by in vitro adhesion to oviductal monolayers.

The specific advantage of BSS in terms of fertilization or cleavage supporting ability may be interpreted in the results of the present study, which show that BSS has a ZP-binding competence that is about threefold compared with that of the control sperm suspension and even more compared with UBSS. Therefore, the superior fertilization competence of selected spermatozoa may be attributable to a higher capability to bind the ZP. However, the different competencies of the three sperm preparations in terms of ZP binding could indicate that this adhesion event acts as a further selection checkpoint for spermatozoa. Indeed, although BSS has a superior ZP-binding competence, the specific advantages of such a sperm subpopulation remain to be determined. As fertilization in the present study was scored as embryo cleavage at $72 \mathrm{~h}$ after insemination, it is possible that either fertilization per se or embryo development, or even both are better supported by BSS. Studies in different species indicating that oviduct adhesion is capable of selecting superior spermatozoa may support all these hypotheses but do not help to distinguish which of them is correct. Finally, as adhesion to the oviduct selects spermatozoa with normal chromatin structure (Ellington et al., 1999) and DNA fragmentation of human spermatozoa is negatively correlated with fertilization outcome (Sun et al., 1997; Lopes et al., 1998; Evenson et al., 1999), it is possible that BSS better supports fertilization and embryo development independently by its oocyte-penetration ability.

As human spermatozoa have been shown to bind both human and bovine Fallopian tube epithelium and to be selected by such an adhesion (Ellington et al., 1998a,b, 1999), future research on adhesion molecules involved in this cell interaction using the bovine model may have 
a profound impact both on the understanding of basic mechanisms involved in early reproductive events and in the development of more physiological methods for sperm selection in reproductive biotechnologies.

This study was supported by a PRIN grant from MIUR.

\section{References}

Abe H, Sendai Y, Satoh T and Hoshi H (1995a) Secretory products of bovine oviductal epithelial cells support the viability and motility of bovine spermatozoa in culture in vitro. Journal of Experimental Zoology 272 $54-61$

Abe H, Sendai Y, Satoh T and Hoshi H (1995b) Bovine oviduct-specific glyco-protein: a potent factor for maintenance of viability and motility of bovine spermatozoa in vitro. Molecular Reproduction and Development 42 226-232

Anderson SH and Killian GJ (1994) Effect of macromolecules from oviductal conditioned medium on bovine sperm motion and capacitation Biology of Reproduction $\mathbf{5 1}$ 795-799

Chian RC and Sirard MA (1995) Fertilizing ability of bovine spermatozoa co-cultured with oviduct epithelial cells Biology of Reproduction $\mathbf{5 2}$ 156-162

Chian RC, Lapointe S and Sirard MA (1995) Capacitation in vitro of bovine spermatozoa by oviduct epithelial cell monolayer conditioned medium Molecular Reproduction and Development 42 318-324

Ellington JE, Carney EW, Farrell PB, Simkin ME and Foote RH (1990) Bovine 1-2-cell embryo development using a simple medium in three oviduct epithelial cell coculture systems Biology of Reproduction $\mathbf{4 3}$ 97-104

Ellington JE, Padilla AW, Vredenburgh EP, Dougherty EP and Foote RH (1991) Behavior of bull spermatozoa in bovine uterine tube epithelial cell coculture: an in vitro model for studying the cell interactions of reproduction Theriogenology 35 977-989

Ellington JE, Evenson DP, Fleming JE, Brisbois RS, Hiss GA, Broder SJ and Wright RW, Jr (1998a) Coculture of human sperm with bovine oviduct epithelial cells decreases sperm chromatin structural changes seen during culture in media alone Fertility and Sterility 69 643649

Ellington JE, Jones AE, Davitt CM, Schneider CS, Brisbois RS, Hiss GA and Wright RW, Jr (1998b) Human sperm function in co-culture with human, macaque or bovine oviductal epithelial cell monolayer Human Reproduction 13 2797-2804

Ellington JE, Evenson DP, Wright RW, Jr, Jones AE, Schneider CS, Hiss GA and Brisbois RS (1999) Higher quality human sperm in a sample selectively attach to oviduct (Fallopian tube) epithelial cells in vitro. Fertility and Sterility 71 924-929

Evenson DP, Jost LK, Marshall D, Zinaman MJ, Clegg E, Purvis K, de Angelis P and Claussen OP (1999) Utility of the sperm chromatin structure assay (SCSA) as a diagnostic and prognostic tool in the human fertility clinic Human Reproduction 14 1039-1049

Eyestone WH and First NL (1989) Co-culture of early cattle embryos to the blastocyst stage with oviducal tissue or in conditioned medium Journal of Reproduction and Fertility 85 715-720

Fazeli A, Duncan AE, Watson PF and Holt WV (1999) Sperm-oviduct interaction: induction of capacitation and preferential binding of uncapacitated spermatozoa to oviductal epithelial cells in porcine species Biology of Reproduction 60 879-886

Gardner DK, Lane M and Batt P (1994) Enhanced rates of cleavage and development for sheep zygotes cultured to the blastocyst stage in vitro in the absence of serum and somatic cells: amino acids, vitamins and culturing embryos in groups stimulates development Biology of Reproduction 50 390-400

Grippo AA, Way AL and Killian GJ (1995) Effect of bovine ampullary and isthmic oviductal fluid on motility, acrosome reaction and fertility of bull spermatozoa Journal of Reproduction and Fertility 105 57-64
Gualtieri R and Talevi R (2000) In vitro-cultured bovine oviductal cells bind acrosome-intact sperm and retain this ability upon sperm release Biology of Reproduction 62 1754-1762

Harper MJK (1994) Gamete and zygote transport. In The Physiology of Reproduction pp 123-187 Eds E Knobil and JD Neill. Raven Press, New York

Hoshi K, Katayose H, Yanagida K, Kimura Y and Sato A (1996) The relationship between acridine orange fluorescence of sperm nuclei and the fertilizing ability of human sperm Fertility and Sterility $\mathbf{6 6} 634$ 639

Hunter RHF (1988) Transport of gametes, selection of spermatozoa and gamete lifespans in the female tracts. In The Fallopian Tubes pp 53-80 Ed. RHF Hunter. Springer-Verlag, New York

Hunter RHF, Fléchon B and Fléchon JE (1991) Distribution, morphology and epithelial interactions of bovine spermatozoa in the oviduct before and after ovulation: a scanning electron microscope study Tissue and Cell 23 641-656

ljaz A, Lambert RD and Sirard MA (1994) In vitro-cultured bovine granulosa and oviductal cells secrete sperm motility-maintaining factor(s) Molecular Reproduction and Development 37 54-60

Lefebvre R and Suarez SS (1996) Effect of capacitation on bull sperm binding to homologous oviductal epithelium Biology of Reproduction $\mathbf{5 4}$ 575-582

Lefebvre R, Chenoweth PJ, Drost M, LeClear CT, MacCubbin M, Dutton JT and Suarez SS (1995) Characterization of the oviductal sperm reservoir in cattle Biology of Reproduction 53 1066-1074

Lopes S, Sun JG, Jurisicova A, Meriano J and Casper RF (1998) Sperm deoxyribonucleic acid fragmentation is increased in poor-quality semen samples and correlates with failed fertilization in intracytoplasmic sperm injection Fertility and Sterility $69528-532$

McNutt T and Killian G (1991) Influence of bovine follicular and oviduct fluids on sperm capacitation in vitro. Journal of Andrology 12 244-252

McNutt T, Olds-Clarke P, Way AL, Suarez SS and Killian G (1994) Effect of follicular or oviductal fluids on movement characteristics of bovine sperm during capacitation in vitro. Journal of Andrology 15 328-336

Martus NS, Verhage HG, Mavrogianis PA and Thibodeaux JK (1998) Enhancement of bovine oocyte fertilization in vitro with a bovine oviductal specific glycoprotein Journal of Reproduction and Fertility 113 323-329

Mermillod P, Vansteenbrugge A, Wils C, Mourmeaux JL, Massip A and Dessy F (1993) Characterization of the embryotrophic activity of exogenous protein-free oviduct-conditioned medium used in culture of cattle embryos Biology of Reproduction 49 582-587

Parrish JJ, Susko-Parrish JL, Winer MA and First N (1988) Capacitation of bovine sperm by heparin Biology of Reproduction 40 1020-1025

Parrish JJ, Susko-Parrish JL, Handrow RR, Sims MM and First NI (1989) Capacitation of bovine spermatozoa by oviduct fluid Biology of Reproduction 40 1020-1025

Paula-Lopes FF, de Moraes AAS, Edwards JL, Justice JE and Hansen PJ (1998) Regulation of preimplantation development of bovine embryos by interleukin-1 Biology of Reproduction 59 1406-1412

Petrunkina AM, Friedrich J, Drommer W, Bicker G, Waberski D and TöpferPetersen E (2001) Kinetic characterization of the changes in protein tyrosine phosphorylation of membranes, cytosolic $\mathrm{Ca}^{2+}$ concentration and viability in boar sperm populations selected by binding to oviductal epithelial cells Reproduction 122 469-480

Pollard JW, Plante C, King WA, Hansen PJ, Betteridge KJ and Suarez SS (1991) Fertilizing capacity of bovine sperm may be maintained by binding to oviductal epithelia cells Biology of Reproduction 44 102107

Smith TT and Yanagimachi R (1990) The viability of hamster spermatozoa stored in the isthmus of the oviduct: the importance of sperm-epithelium contact for sperm survival Biology of Reproduction 42 450-457

Sun JG, Jurisicova A and Casper RF (1997) Detection of deoxyribonucleic acid fragmentation in human sperm: correlation with fertilization in vitro. Biology of Reproduction $\mathbf{5 6}$ 602-607

Talevi R and Gualtieri R (2001) Sulfated glycoconjugates are powerful modulators of bovine sperm adhesion and release from the oviductal epithelium in vitro. Biology of Reproduction 64 491-498 
Thomas PGA, Ball BA and Brinsko SP (1994) Interaction of equine spermatozoa with oviduct epithelial cell explants is affected by estrous cycle and anatomic origin of explant Biology of Reproduction $\mathbf{5 1}$ 222-227

Thomas PGA, Ball BA and Brinsko SP (1995) Changes associated with induced capacitation influence the interaction between equine spermatozoa and oviduct epithelial cell monolayers Biology of Reproduction Monographs 1 697-705

Van Dyk Q, Lanzendorf S, Kolm P, Hodgen GD and Mahony MC (2000) Incidence of aneuploid spermatozoa from subfertile men: selected with motility versus hemizona-bound Human Reproduction 15 1529-1536
Verhage HG, Fazleabas AT, Mavrogianis PA, O'Day-Bowman MB, Donnelly KM, Arias EB and Jaffe RC (1997) The baboon oviduct: characteristics of an oestradiol-dependent oviduct-specific glycoprotein Human Reproduction Update 3 541-552

Received 30 July 2002.

First decision 19 September 2002.

Revised manuscript received 11 October 2002

Accepted 18 October 2002. 